lability, onset of urinary incontinence and generalised seizures, loss of mobility, loss of self-care skills and behavioural difficulties was evident. Six months prior to her death she was transferred to a nursing home where she was bedridden, required a permanent urinary catheter and needed 24-hour nursing care. She died from bronchopneumonia. Typical clinical features of DS were present. Prior to the onset of deterioration the patient was in good health and functioned at a moderate level of learning disability. Her full blood count, profile and thyroid hormone results were generally normal throughout her illness although macrocytosis was present for the last three years and borderline hypothyroidism was treated with thyroxine replacement two years prior to death. A clinical diagnosis of Alzheimer's disease was made. There was no family history of dementia although one sister required treatment for depression. The patient's karyotype was $46, \mathrm{XX},-21,+\mathrm{t}$ (2lq21q).

Genetic aspects of ATD have recently aroused considerable interest with particular reference to apolipoprotein $\mathrm{E}$ genotype and location of the gene coding for amyloid precursor protein on chromosome 21 (Goate et al, 1989). It is possible that further significant advances could be made by investigating the occurrence of ATD in uncommon forms of DS. Molecular genetic studies of these rare cases is recommended as they could aid determination of genetic loci predisposing to Alzheimer's disease.
Goate, A. M.. Haynes, A. R.. Owen, M. J., el al (1989) Predisposing locus for Alzheimer's disease on chromosome 21. Lancet, i. 352-355.

Oliver, C. \& Holland, A. J. (1986) Down's syndrome and Alzheimer's disease: review. Psychological Medicine, 16, 307322.

Prasiler. V. P. (1993) Presenile dementia associated with unbalanced Robertsonian translocation form of Down's syndrome. Lancet, 342, 686-687.

Rowe, I. F., Ridler, M. A. C. \& Gibberd, F. B. (1989) Presenile dementia associated with mosaic trisomy 21 in a patient with Down syndrome child. Lancet, ii, 229.

Sylvistr. P. E. (1986) The anterior commissure in Down's syndrome. Journal of Mental Deficiency Research, 13, 19-26.

V.P. PRASHER

University of Birmingham

Birmingham B15 2QZ

\section{Train of thought}

SIR: I am not a train spotter, but given my interest in Asperger's syndrome, I am a train spotter spotter. I note that many of my colleagues are also train spotter spotters, which makes me a train spotter spotter spotter. I have not yet met any other train spotter spotter spotters but $I$ have started a list just in case and will write down their numbers if I can.

Institute of Psychiatry

R. GoOdman 